\title{
Machining of complex cross-sectional profile parts based on NC ZHOU Yong-qing ${ }^{1, a}$, WEI Bo ${ }^{2, b}$, XUE Shao-ye ${ }^{3, c}$ \\ ${ }^{1,2,3}$ Department of Mechanical and Electrical Engineering, Henan Quality Polytechnic, Ping dingshan, 467000, China, \\ a,b,c zlxy2009@126.com
}

Keywords: Complex cross-sectional profile;Curve fitting method;Minimum condition

\begin{abstract}
In order to achieve the manual programming of complex cross-sectional profile parts, the use of curve fitting, use mapping software to draw the outline of the curve, the contour is segmented, with minimum condition arc or straight line to fit the line segment to give the arc endpoint coordinates and the radius, or the coordinates of the endpoints of a straight line, Finally, with the simple linear or circular instruction, the preparation of a machining program.
\end{abstract}

\section{Introduction}

The irregular curve, which is a type of curve with a series of value points connected with smooth curve to, and can't be described by mathematical formula. For rotational parts with irregular curve profile, its machining process is difficult, the ordinary lathe is difficult to guarantee the machining precision and efficiency. For $\mathrm{CNC}(\mathrm{Computer}$ Numerical Control), it has the following two difficulties: on the one hand, if we adopt the manual programming, even though we know the coordinates of points, but there are no available interpolation methods, so the machining procedures can't be carried out $[1,2]$. On the other hand, if we adopt the automatic programming, we can develop the machining programs, but the preparation of the program is too more complicated to use the combined-cycle instruction. Besides, the empty machining also affects the machining accuracy and efficiency. To solve the above problems, we need to segment the contour curve according to the change of curvature, and to use the arc and line to fit different segments. Through the calculation, we can get the minimum arc and straight line to fit the segments, and calculate the circular endpoint coordinates and radius by using the drawing methods. Finally, we develop the NC program. This method can simplify program and improve machining efficiency [3-5].

\section{The basic principles of the irregular curve fitting}

Setting the coordinates of a series of points of irregular curves are: I (y1i, y2i), i=1, 2, 3, ., n.

We need to use a circular arc $\left(y_{1}-A\right)^{2}+\left(y_{2}-B\right)^{2}=R^{2}$ to fit, and to make complex curve with arc the approximation, which can make the radius deviation from points to the center distance is the smallest. Obviously, if the point I (y1i, y2i) on the arc $\left(y_{1}-A\right)^{2}+\left(y_{2}-B\right)^{2}=R^{2}$, there is no deviation, that is $\left(y_{1}^{\prime}-A\right)^{2}+\left(y_{2}^{\prime}-B\right)^{2}=R^{2}$. If the type value of the point is not on the arc $\left(y_{1}-A\right)^{2}+\left(y_{2}-B\right)^{2}=R^{2}$, there is a deviation, the deviation can be defined as:

$$
\Delta_{i}=\sqrt{\left(y_{1}^{i}-A\right)^{2}+\left(y_{2}{ }^{i}-B\right)^{2}}-R \quad \text { (1) }
$$

In order to eliminate the influence of deviation of plus or minus, we use the sum of squares $Q$ as the overall error.

$$
\left.Q(A B, R)=\sum_{i=1}^{n} \Delta_{i}^{2}=\sum_{i=1}^{n} \sqrt{\left(y_{1}^{i}-A\right)^{2}+\left(y_{2}^{i}-B\right)^{2}}-R\right)^{2}
$$

So calculating the problem of fitting arc is that according to (2) calculated Q for the minimum A, $\mathrm{B}$, and R. A, B and R are determined by the following equations [10-12]. 


$$
\left\{\begin{array}{l}
\frac{\partial Q(A, B, R)}{\partial A}=0 \\
\frac{\partial Q(A, B, R)}{\partial B}=0 \\
\frac{\partial Q(A, B, R)}{\partial R}=0
\end{array}\right.
$$

Calculating the equation of $\mathrm{A}, \mathrm{B}$ and $\mathrm{R}$, the spatial location of fitting round is determined, the error of fitting arcs and curves through (1) computing, the maximum error at the end points, appropriate to reduce the number of dots that participation in fitting calculation, we can reduce the error to the extent range.

If we use the arc fitting, when change in curvature of the curve is little, we can achieve a good fit with a few arcs. When the curvature of the curve has changed so much, the curve is divided into several sections to increase the fitting quantity, and also can achieve the best approximation curve.

\section{The CNC machining instance}

In this article, we take the lathe of the cutter disc of a molding tool as an example to describe the machining process. Axis cross-sectional profile of the cutter disc of a molding tool consists of 198 points with smooth curves connected together. In order to facilitate the preparation of NC machining program to translate each type point coordinate, we build the machine coordinate system as shown in Fig. 1.

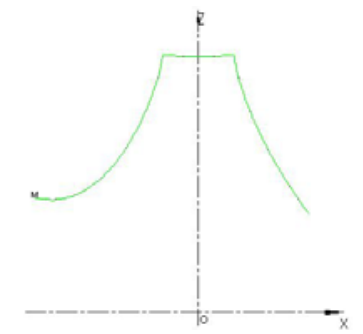

Fig.1. The machining coordinate system

\subsection{The fitting calculation}

For the curve segment, based on the curvature of the contour curve changes. For the left curve, for the curvature changes, the curve to be divided into four sections. For the right curve, for the curvature of the less obvious changes, the curve to be divided into two sections [6-9].

Fitting calculation in the first paragraph of the left: This is a total of 28 data points, substituting (2), according to (3) presentation of the equations to solve the fitting radius $\mathrm{R} 1=34.586$, the center coordinates $(-136.371,360.925)$.

Fitting calculation in the Second paragraph of the left: This is a total of 18 data points, substituting (2), according to (3) presentation of the equations to solve the fitting radius $\mathrm{R} 1=74.268$, the center coordinates $(-188.055,421.150)$.

Fitting calculation in the third paragraph of the left: This is a total of 15 data points, substituting (2), according to (3) presentation of the equations to solve the fitting radius $\mathrm{R} 1=144.548$, the center coordinates $(-255.177,465.945)$.

Fitting calculation in the fourth t paragraph of the left: This is a total of 19 data points, substituting (2), according to (3) presentation of the equations to solve the fitting radius $\mathrm{R} 1=66.102$, the center coordinates (-163.810, 433.684).

Fitting calculation in the first paragraph of the right: This is a total of 36 data points, substituting (2), according to (3) presentation of the equations to solve the fitting radius $\mathrm{R} 1=203.337$, the center coordinates $(417.807,534.885)$.

Fitting calculation in the Second paragraph of the right: This is a total of 38 data points, substituting (2), according to (3) presentation of the equations to solve the fitting radius $\mathrm{R} 1=103.447$, the center coordinates $(236.837,449.848)$. 
The middle section has a total of 44 data points, considering the precision machining, we use a straight line fitting.

When we determining the RADIUS and center coordinate, we determine the coordinate position of the arc. To test segment reasonable and fitting accuracy, we import AUTCAD software to generate a standard cutter profile curve after a coordinate translation of data points. Then, we draw the arcs by the center and the radius in the same plane, after pruning, we get the arc as shown in Fig. 2. By observing and measuring, we can see the more obvious deviations in the first Sec paragraph of the left and the second paragraph of the right, other segment deviation is not obvious. For the processing of the cutter disc, the deviation within the allowable range, be seen in this article section is basically reasonable.

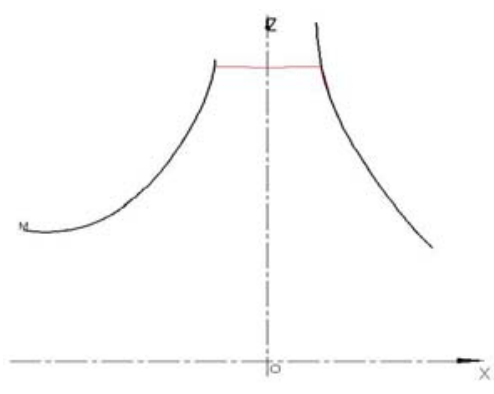

Fig.2. The arc fitting curve

When we know the Center and radius of the arc, but still cannot meet the NC processing programming needs, would also like to know the coordinates of both ends of the arc. The coordinates of both ends of the arc in two ways, A column equations, calculated in turn obtained the coordinates of the intersection of two adjacent arc is the endpoint, Computation is relatively large. the other is the drawing, using CAD software to draw each arc, by marking measurements obtained endpoint coordinates, this method is simpler, this article on the use of this method. Through drawing, get the endpoint coordinates are as follows:

The left five endpoints coordinates are : $(-149.721,293.053),(-91.297,308.454),(-58.875$, $347.835)(-39.733,388.045)(-32.987,414.624)$.

The right three endpoint coordinates are: $(100.960,279.953),(54.896,351.369),(32.987$, 414.624).

\subsection{The CNC programming and machining}

\subsubsection{Machining process programming}

Analysis the Machining process planning firstly. This part is a typical disk part which can be done on a common CNC machine tool. The selection of fixture is the three grasping chucks, the tools is the general lathe tool, the machining can be divided into two processes, after the end of the processing is completed, leaving a certain extended the length of the positioning, Through the above calculation, the processing parameters are enough, we use the composite cycle instruction G71 to complete the programming. Then we need to select the tool and fixture. For the tool: No. 1 tool is $90^{\circ}$ cylindrical knife, knife sharp corners of $55^{\circ}$; No. 2 tool is cutter blades, knife sites in the left corner, blade width $3 \mathrm{~mm}$. For the fixture:using three-jaw chuck.

Finally, we need to determine the processing planning.

The programs are as follows:

O0001

N10 T0101

N50 G71 P60 Q120 U0.2 W0.2 F0.2 
N190 M30

\subsubsection{The actual processing}

After the simulation by Yulong CNC system, the program has not problem, then process it directly on the machine. The results of processing are shown in Fig.3. ( Note: the actual process without processing the center bore, the main processing object is the tool profile ).

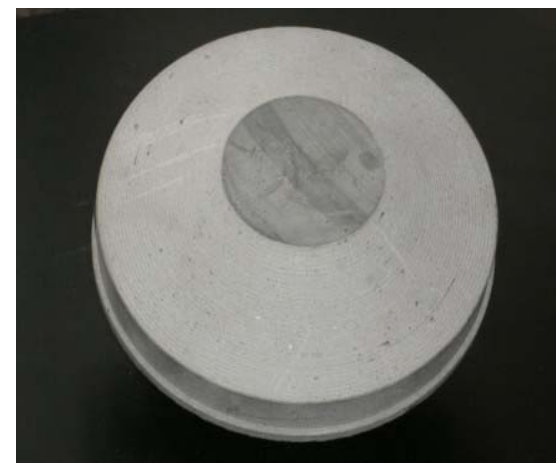

Fig.3. the actual processing of the workpiece

\section{Conclusion}

For the disc parts with the irregular curve, we use arc to fit the contour curve by segmentation fitting method. The method make the machining of the complex disc parts is easy to achieve. In this way, the manual programming simplifies the preparation of the program and improves the efficiency of processing. The method can be applied in the process machining other complex parts, and has some practical value.

\section{References}

[1] WEI Guo-zhe, LIU Kang, LEI Guang-qiang,WANG Yu-chun ,Research on Approximation Algorithm of Spline Curve in AutoCAD. Mechanical Engineering \& Automation, no.03 (2010), p.192-193.

[2] LIU Xiang-hong, Machining Method of Free Plane-curve Based on Manual Programming. Machinery \& Electronics, no. 11 (2009), p.75-77.

[3] PEI Xing-lin, Research on NURBS Interpolation of Combined Surface. Science Technology and Engineering, no.01 (2010), p.287-290.

[4] SHAN Dongri, Research on NC Machining of Plane Parametric Curve Based on Difference Interpolation of Conic Spline[J]. Manufacturing Technology \& Machine Tool, no.02 (2009), p.95-98.

[5] WANG Zhi-ming,LI Jian-ping, Programming of Formula Curve for CNC Lathe. Equipment Manufacturing Technology, no.07(2009), p.138-139.

[6] Huang Guiqin,Du Zhiqiang,Sun Xiaolao, Study on NC Programming Approach of the Listing Point Cam Curve. Journal of Mechanical Transmission, no.04(2009), p. 116-117,122.

[7] XUE Dong-bin, WANG Yan-lin, SONG Xiao, YANG Wen-zheng, Application of MasterCAM on Numerical Control Machining Programme of Spacial Curve. Manufacture Information Engineering of China, no.11(2006), p. 34-35.

[8] YUE Ying,HAN Qingyao,WANG Zhangqi, An Approaching Method for NURBS Curve in NC Machining. Machine Tool \& Hydraulics, no.01(2007), p. 63-64+121.

[9] XU Long,JI Hanqi, Programming for the CNC Lathe of Non-circular Curve in Mastercam. New Technology \& New Process, no.08(2008), p. 16-17+1. 